\title{
Tension Pneumocephalus Related to Spontaneous Skull Base Dehiscence in a Patient on BiPAP
}

\author{
Wannemuehler, Todd J.; Hubbell, Richard D.; Nelson, Rick F. \\ *Department of Otolaryngology-Head and Neck Surgery, Indiana University School of Medicine, \\ Indianapolis, Indiana \\ †Department of Otolaryngology-Head and Neck Surgery, Loyola University School of Medicine, \\ Chicago, Illinois
}

Address correspondence and reprint requests to Rick F. Nelson, M.D., Ph.D., 355 W. 16th Street, Suite 3200, Indianapolis, IN, 46202; E-mail:

ricnelso@iupui.edu

There is no funding to disclose for this manuscript.

IRB Attestation: "Case studies which involve only one subject are not considered to be systematic investigations, and do not require IRB approval”; from:

http://researchcompliance.iu.edu/hso/hs_level_review.html

The authors have no financial relationships.

The authors have no conflicts of interest to disclose.

\begin{abstract}
Spontaneous pneumocephalus is an uncommon phenomenon that may develop in patients with occult skull base defects. There have been reports of pneumocephalus occurring spontaneously in the setting of continuous positive airway pressure (CPAP) use (1). Tension pneumocephalus represents a neurosurgical emergency where intracranial air is trapped with increasing pressures resulting in
\end{abstract}

This is the author's manuscript of the article published in final edited form as:

Wannemuehler, T. J., Hubbell, R. D., \& Nelson, R. F. (2016). Tension Pneumocephalus Related to Spontaneous Skull Base Dehiscence in a Patient on BiPAP: Otology \& Neurotology, 37(9), e322-e324.

https://doi.org/10.1097/MAO.0000000000001141 
neurological deterioration (2). Previous literature has also documented the growing understanding of how obesity, elevated intracranial pressure (ICP), obstructive sleep apnea (OSA), and cortical skull thinning are associated with spontaneous tegmen dehiscence and cerebrospinal fluid (CSF) leakage (3). Other mechanisms for spontaneous CSF leak include aberrant arachnoid granulation, congenital skull base dehiscences, increased abdominal and thoracic pressure resulting in reduced cerebral venous drainage, and age-related cortical thinning. In this report, we present the case of a bilevel positive airway pressure (BiPAP) user with an undiagnosed spontaneous tegmen dehiscence who developed spontaneous tension pneumocephalus.

\section{CASE REPORT}

An obese (body mass index [BMI] = 31.5 kg/m2) 63-year-old man with well-controlled insulindependent diabetes, hypertension, hypothyroidism, and OSA (Apnea Hypopnea Index 22) experienced progressive anosmia over a week and the following week developed new onset severe headaches. He consistently used a BiPAP machine (Exhalational PAP 6-15 cm H2O, pressure support 0-20 cm H2O) to treat his OSA. One week later, he was traveling alone out of town when he developed severe diarrhea and emesis. The next day, family members noted confused speech content during phone contact and therefore arranged for emergency medical personnel to evaluate the patient. The patient was found incoherent and taken to an outside hospital emergency department where he met systemic inflammatory response syndrome criteria with altered mental status, agitation, tachycardia, massive headache, and lab results consistent with diabetic ketoacidosis.

Head magnetic resonance imaging (MRI) and computed tomographic (CT) examination demonstrated classic tension pneumocephalus and tegmen dehiscence on the right (Fig. 1, A-C). The anterior skull base was intact (images not shown). The patient had no reported previous history of rhinorrhea, neurosurgical procedures, or significant head trauma. He underwent a right temporal craniotomy with extradural and intradural repair of the defect with autologous bone, muscle, and 
pericranial grafts; no postoperative lumbar drain was used. The patient was eventually discharged home and discontinued use of his BiPAP.

One month later, the patient continued to have a large subcutaneous air collection at the surgical site and repeat CT scanning demonstrated air tracking through persisting dehiscent areas of the right tegmen intracranially and into the extracranial soft tissues. Squamous temporal bone measurements using techniques outlined by a previous study (3) demonstrated cortical skull thinning with an average thickness of $2.27 \mathrm{~mm}$ (Fig. 1D); this value is within the confidence interval of similar spontaneous CSF leak patients in that study $(3.29 \pm 0.68 \mathrm{~mm}$ in obese spontaneous CSF leak patients versus $4.25 \pm 0.58 \mathrm{~mm}$ in obese patients without CSF leak; $\mathrm{p}<0.0001$ ). The patient subsequently underwent revision craniotomy where persisting tegmen and dural defects without encephalocele were noted and repaired; again no postoperative lumbar drain was used. Postoperative CT 3 weeks later showed complete resolution of pneumocephalus (Fig. 2A) and satisfactory bone grafting of the skull base (Fig. 2B). Six months after this revision, the patient has resumed BiPAP use (Exhalational PAP 6-15 cm H2O, Pressure Support 0-15 cm H2O) without recurrent pneumocephalus. Persistent anosmia is the only long-term sequelae the patient has experienced.

\section{DISCUSSION}

This case is the first reported case of tension pneumocephalus in a patient with a skull base dehiscence and likely CSF leak related to use of BiPAP. Spontaneous CSF leaks are increasing in prevalence (4) and are associated with obesity and OSA. We think one potential mechanism for the development of spontaneous CSF leaks is increased ICP during apnea episodes in OSA. Imaging features of chronically elevated ICP include empty sella, enlarged Meckels’ cave, and papilledema. Interestingly, many patients with spontaneous CSF leaks do not demonstrate these image findings. It is possible that transient spikes in ICP during apneas may play a role in spontaneous CSF leaks. While the exact pathophysiology of spontaneous CSF leaks is unknown, we have previously documented a relationship between spontaneous CSF leaks, OSA, and thinning of the calvarium (3) and 37\% of patients with 
spontaneous CSF leaks had OSA (4). We think that it is important to raise awareness of the relationship between these factors because as the incidence of obesity and OSA continue to rise, so too will the rate of associated conditions such as spontaneous CSF leaks and pneumocephalus. The fact that even minimal PAP settings result in supraphysiologic middle ear pressure places these patients at risk of developing tension pneumocephalus (5). It is also important to realize that surgical repair of the skull base may allow for CPAP/BiPAP resumption in OSA. As many as 50\% of patients have been found to have multiple skull base defects. Thus, even with successful surgical repairs, it is important to recognize that these patients remain at risk of developing recurrent pneumocephalus from secondary sites of dehiscence and CSF leaks. 


\section{REFERENCES}

1. Jarjour NN, Wilson P. Pneumocephalus associated with nasal continuous positive airway pressure in a patient with sleep apnea syndrome. Chest 1989; 96:1425-1426.

2. Pulickal GG, Sitoh YY, Ng WH. Tension pneumocephalus. Singapore Med J 2014; 55:e46-e48.

3. Nelson RF, Hansen KR, Gantz BJ, et al. Calvarium thinning in patients with spontaneous cerebrospinal fluid leak. Otol Neurotol 2015; 36:481-485.

4. Nelson RF, Gantz BJ, Hansen MR. The rising incidence of spontaneous cerebrospinal fluid leaks in the United States and the association with obesity and obstructive sleep apnea. Otol Neurotol 2015; 36:476480.

5. Thom JJ, Carlson ML, Driscoll CL, et al. Middle ear pressure during sleep and the effects of continuous positive airway pressure. Am J Otolaryngol 2015; 36:173-177. 

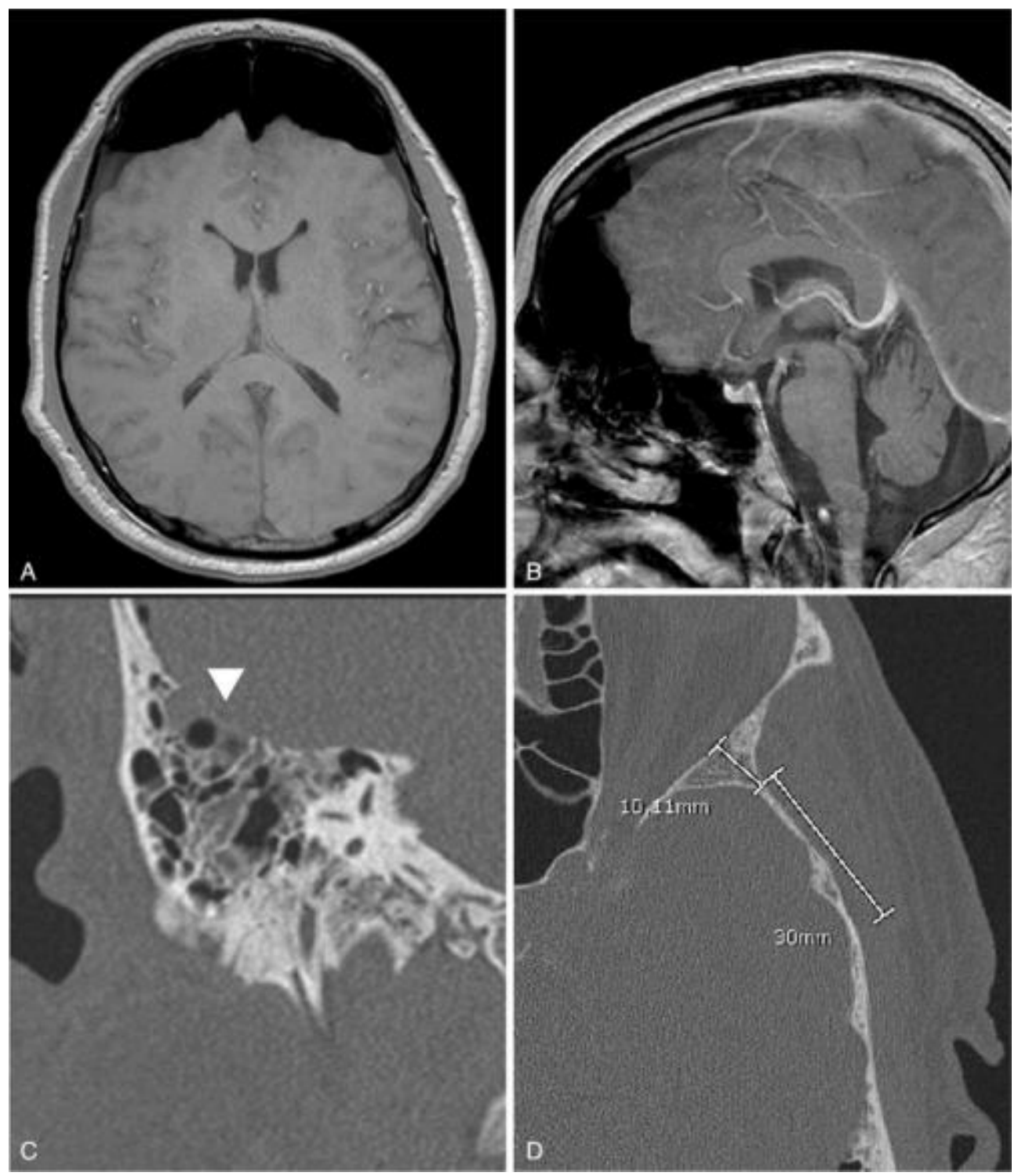

FIG. 1. MRI and CT images of the brain of our patient at presentation. (A), T1-weighted axial MRI image demonstrates tension pneumocephalus of the frontal lobes displaying the classic Mount Fuji sign. (B), Sagittal T1-weighted image demonstrating tension pneumocephalus with compression of the frontal lobes. (C), Coronal non-contrast CT image demonstrates bony tegmen dehiscence overlying the right mastoid cavity. (D), Axial non-contrast CT images marked with measurements used to calculate calvarial thickness. Measurements demonstrated severe calvarial thinning. CT indicates computed tomographic; MRI, magnetic resonance imaging. 


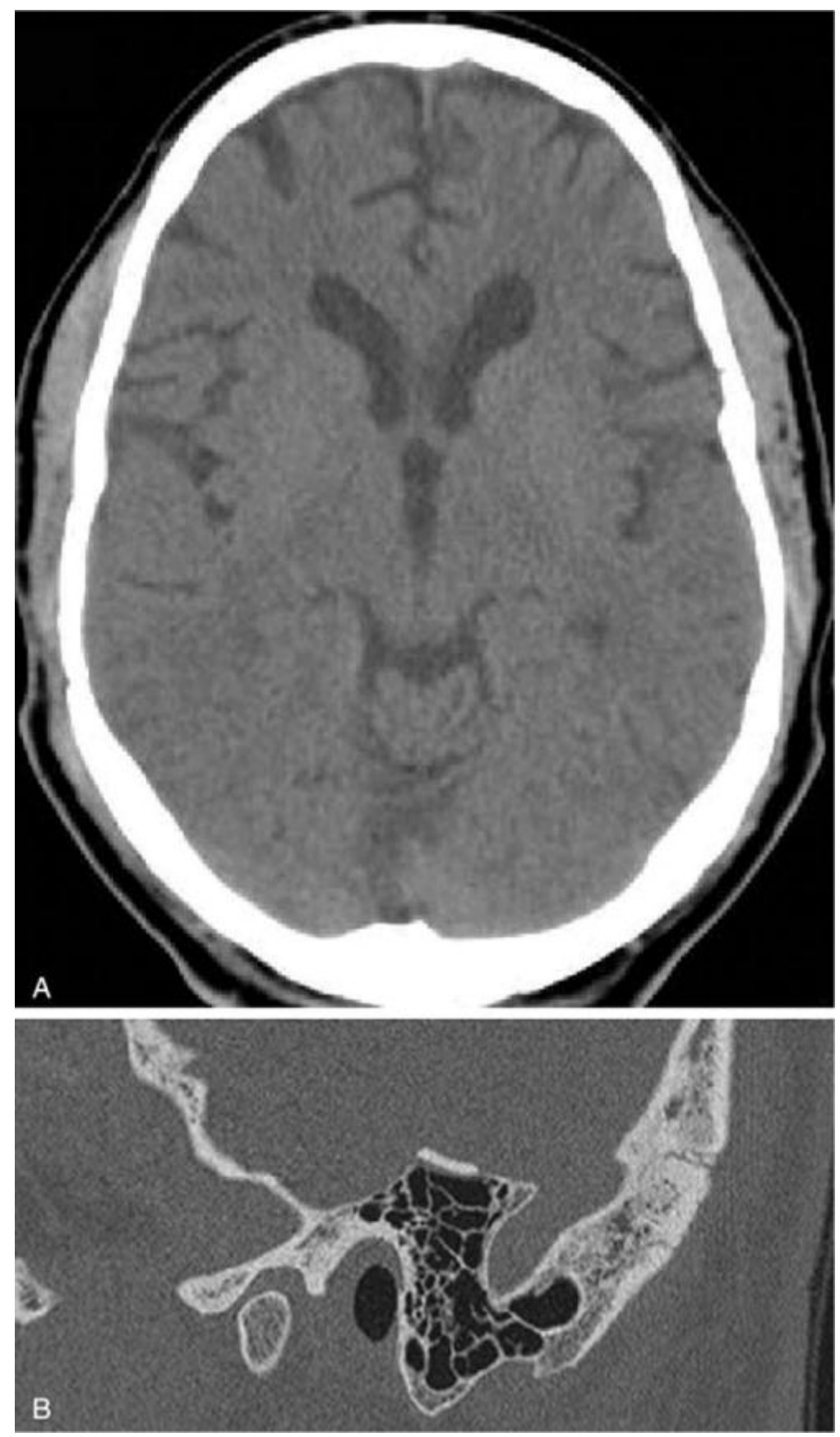

FIG. 2. CT images after revision right-sided craniotomy for repair of CSF leak and resuming use of BiPAP. (A), Axial non-contrast head CT demonstrates complete resolution of pneumocephalus. (B), Parasagittal non-contrast CT demonstrating prior bony repair using autologous squamous temporal bone. BiPAP indicates bilevel positive airway pressure; CSF, cerebrospinal fluid; CT, computed tomographic. 